\title{
GLI ACCELERATORI NUCLEARI NELLA TERAPIA DEI TUMORI
}

\author{
DOMENICO SCANNICCHIO (*)
}

Nota presentata dal m.e. Ferdinando Borsa

(Adunanza del 24 Maggio 2012)

SuNTO. - Vengono riportati in forma schematica i principali vantaggi dell'adroterapia rispetto alla radioterapia convenzionale. Dopo aver passato in rassegna le principali caratteristiche degli acceleratori di particelle vengono descritte le attrezzature del Centro Nazionale di Adroterapia Oncologica, in particolare i sistemi di posizionamento del paziente e di rilascio della dose di radiazione.

$* * *$

ABSTRACT. - The main advantages of hadrontherapy against the traditional radiotherapy are reported. After a brief review of particles accelerators, the facilities of the 'Centro Nazionale di Adroterapia Oncologica' (National Center of Oncological Hadrontherapy) in Pavia are described with details on the patient positioning and beam irradiation systems.

\section{INTRODUZIONE}

Da oltre 60 anni vengono sviluppati sistemi di accelerazione di particelle elettricamente cariche per impiegarle come proiettili al fine di indagare la struttura della materia (atomi, nuclei e particelle elementari). Solo da circa 40 anni questi fasci di particelle vengono impiegati a scopo terapeutico, in particolare per distruggere cellule neoplastiche (tumori).

(*) Dipartimento di Fisica, Università degli Studi di Pavia, Italy.

E-mail: domenico.scannicchio@pv.infn.it 
Prima di descrivere tale applicazione degli acceleratori è opportuno prendere in considerazione le particelle utilizzate come proiettili. Come noto la materia è formata da atomi e/o da strutture costituite da molti atomi diversi (molecole e macromolecole), con ciascun atomo costituito da una nuvola di elettroni che circonda un nucleo centrale, centomila volte più piccolo dell'atomo stesso, dotato di una carica elettrica contraria a quella degli elettroni orbitanti (l'atomo normalmente è elettricamente neutro) e contenente quasi tutta la massa dell'atomo.

I proiettili prodotti dagli acceleratori sono le particelle citate, gli elettroni (di massa molto piccola) e quelle costituenti i nuclei: protoni e neutroni (2000 volte più massicci degli elettroni) oppure i nuclei stessi, chiamati ioni essendo dotati di carica elettrica (positiva). Oltre a queste particelle gli acceleratori permettono la formazione di fasci di radiazione elettromagnetica, cioè raggi $\mathrm{X}$ e raggi gamma di energia molto elevata.

Per comprendere l'efficacia di particelle e radiazioni X nel distruggere le cellule tumorali è indispensabile conoscere in che modo esse interagiscono con la materia in cui si propagano, diminuendo gradualmente la propria energia iniziale che viene rilasciata al materiale stesso con molteplici processi in successione (processi secondari). Si definisce dose assorbita il rapporto tra l'energia assorbita dal materiale e la massa del materiale, la cui unità di misura nel Sistema Internazionale è il gray $(\mathrm{Gy})$, corrispondente ad un joule per chilogrammo. Radiazioni X e particelle (elettroni, protoni e ioni) rilasciando energia nel materiale gradualmente perdono energia fino ad arrestarsi: la distanza media percorsa si chiama range ed è tanto più elevato quanto maggiore è l'energia iniziale (Fig. 1, dove il mezzo attraversato è l'acqua, principale costituente del materiale biologico).

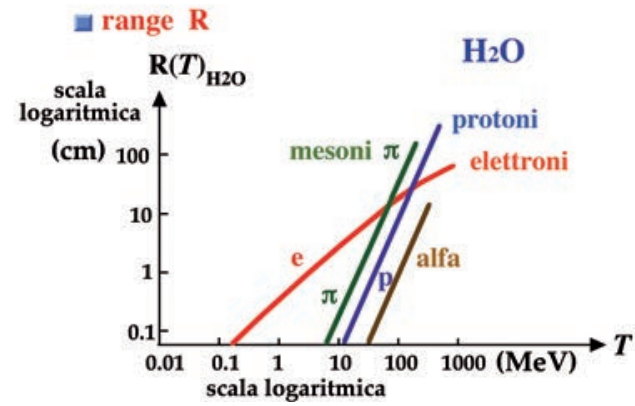

Fig. 1 - Viene riportato il percorso medio (range) in acqua di vari tipi di particelle.

La cura dei tumori mediante fasci di particelle o radiazioni X è chiamata radioterapia. Quella convenzionale impiega raggi X ed elettro- 
ni. La radioterapia che impiega particelle nucleari (protoni o nuclei stessi) è detta adroterapia (le particelle nucleari sono chiamate adroni, cioè particelle che interagiscono fra loro con una forza attrattiva enorme, chiamata interazione nucleare forte).

L'adroterapia presenta in generale 3 vantaggi relativamente alla radioterapia convenzionale: elevato rilascio di energia a fine percorso, collimazione del fascio nel penetrare la materia, modalità di rilascio dell'energia alle macromolecole presenti nelle cellule, in particolare al DNA. Vediamo ora i dettagli di queste 3 caratteristiche vantaggiose.

\section{RILASCIO DI ENERGIA NEL MATERIALE ATTRAVERSATO}

In Fig. 2 sono riportati i rilasci di energia da parte di varie radiazioni (corpuscolari e raggi $\mathrm{X}$ ) man mano esse penetrano nel materiale (acqua): come si può facilmente rilevare i protoni rilasciano gran parte della loro energia a fine percorso (picco di Bragg), mentre raggi $\mathrm{X}$ e gamma ed elettroni rilasciano l'energia prevalentemente all'ingresso del materiale. Calibrando l'energia dei protoni l'energia può essere concentrata in una determinata regione danneggiandola, contrariamente alle altre radiazioni che danneggiano il materiale prevalentemente all'ingresso con limitata distruzione del bersaglio in profondità. Questa caratteristica dei protoni (picco di Bragg) viene ulteriormente accentuata nel caso di radiazioni costituite da nuclei (ad esempio di ioni di carbonio o di ossigeno). In pratica gli adroni agiscono in modo simile $\mathrm{a}$ bombe di profondità nel materiale.

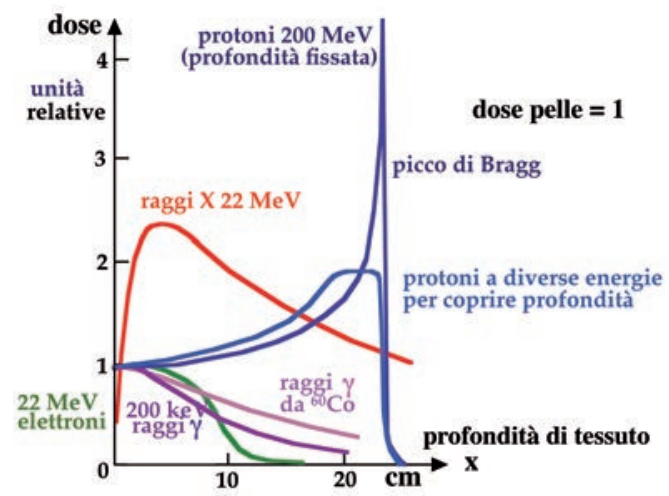

Fig. 2 - Rilascio di energia lungo il percorso in acqua da parte di varie particelle. Per i protoni monoenergetici è evidente il picco di Bragg. 


\section{Collimazione Del Fascio}

In Fig. $3 a$ e $3 b$ sono mostrate le sezioni di fasci di elettroni, raggi X, protoni e ioni carbonio. A partire da un fascio di date dimensioni (rettangolo iniziale) gli elettroni già a $10 \mathrm{~cm}$ di profondità si sono allargati in quello che è chiamato uno sciame elettromagnetico (3a) e analogamente i raggi $\mathrm{X}$ a $15 \mathrm{~cm}$ di profondità ( $3 \mathrm{~b}$ ) mentre i protoni e gli ioni carbonio (la cui energia comporta una profondità di fine percorso di $15,5 \mathrm{~cm}$ ) alle diverse profondità restano molto collimati. Nella pratica ciò significa che elettroni e radiazione $\mathrm{X}$ e gamma causano distruzioni lateralmente al fascio, dove il materiale biologico se sano deve subire il minor danno possibile e se vitale per l'organismo non deve assolutamente essere danneggiato.

La particolare elevata collimazione del fascio di adroni permette di "centrare" il bersaglio con elevata precisione (dell'ordine di un millimetro), precisione che tuttavia richiede un posizionamento del paziente dal punto di vista tecnico assai complesso, come vedremo nel seguito, trattandosi di un organismo vitale e non di un corpo inerte.
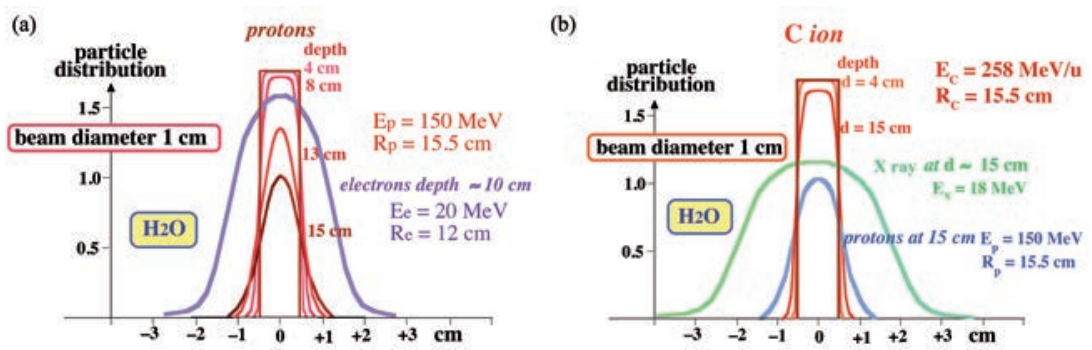

Fig. 3 - a) Sezioni di un fascio di protoni in acqua a varie profondità paragonate alla sezione di un identico fascio di elettroni alla profondità di $12 \mathrm{~cm}$. Si osservi la maggiore collimazione del fascio di protoni rispetto agli elettroni che diffondono lateralmente. b) Sezioni di un fascio di ioni carbonio a varie profondità paragonate con la sezione di un fascio identico di raggi X: la collimazione degli ioni carbonio è molto elevata.

\section{ModALITÀ DI RILASCIO DELL'ENERGIA}

Quando una radiazione (corpuscolare o raggi X) attraversa la materia, in particolare la materia biologica, rilascia energia agli atomi e molecole del mezzo: questa interazione con le strutture molecolari comportano soprattutto la rottura di legami chimici. Quando la macromolecola coinvolta è il DNA si hanno rotture di legami che spesso il DNA riesce ad autoriparare. 
La grandezza fisica che rappresenta il rilascio di energia per unità di percorso è chiamato LET (Linear Energy Transfer). Le varie radiazioni presentano un LET diverso: mentre raggi $\mathrm{X}$, raggi gamma, elettroni sono radiazioni a basso LET, cioè creano pochi danni per unità di percorso, gli adroni (protoni ma soprattutto ioni) hanno un LET elevato creando un tale numero di danni (rotture di legami chimici) da rendere in particolare la macromolecola DNA irreparabile. La distruzione del DNA per una cellula significa l'impossibilità di replicarsi e quindi la sua morte.

In realtà le macromolecole nelle cellule si trovano immerse in un ambiente acquoso e le radiazioni a basso LET provocano la rottura delle molecole d'acqua in due frammenti dotati di elevata energia (acquisita dall'interazione con la radiazione) chiamati radicali liberi. Questi radicali liberi sono molto reattivi e interagiscono chimicamente con le macromolecole presenti nei dintorni: questa interazione chimica danneggia le macromolecole causando la rottura di legami chimici, legami che il DNA per sua natura è in parte in grado di riparare. Dunque le radiazioni a basso LET provocano un danno di tipo chimico al materiale biologico.

Nel caso della radiazione ad alto LET, oltre al danno chimico, l'elevato numero di rottura di legami nelle macrostrutture (DNA) provoca un danno di tipo fisico, praticamente "tagliando" la macromolecola in modo non riparabile. In effetti quando con un coltello affilato si taglia la carne, l'azione di "tagliare" comporta la rottura di innumerevoli legami chimici (dell'ordine del numero di Avogadro).

In conclusione l'efficacia distruttiva di cellule è assai maggiore per la radiazione costituita da adroni rispetto alle radiazioni della radioterapia convenzionale: per gli adroni il danno fisico irreparabile si aggiunge a quello chimico da radicali liberi. In Fig. 4 sono mostrate delle simulazioni di danni determinati da diverse radiazioni, nella scala del DNA mostrato a sinistra. L'efficacia dell'adroterapia si evidenzia in modo particolare nel caso di tumori radio-resistenti alla terapia convenzionale.

\section{Piani Di TRATTAMENTO}

Per eseguire un irraggiamento è indispensabile predisporre un piano di trattamento che viene sviluppato a partire dalle immagini radiografiche e tomografiche che forniscono la posizione e la forma tridimensionale del tumore e dei tessuti circostanti, dalla natura delle cellule neoplastiche e dalla prossimità di organi vitali. Il piano di trattamento definisce la dose da impartire in ciascun irraggiamento, i para- 
metri del fascio (energia, intensità, dimensione), le direzioni di irraggiamento (definite campi) e il numero di irraggiamenti. Il piano di trattamento viene messo a punto dalla collaborazione del medico radioterapista e del fisico sanitario. Tutti i relativi dettagli vengono inseriti in un sistema di calcolo che provvede alla esecuzione del piano, come descritto nei paragrafi successivi.
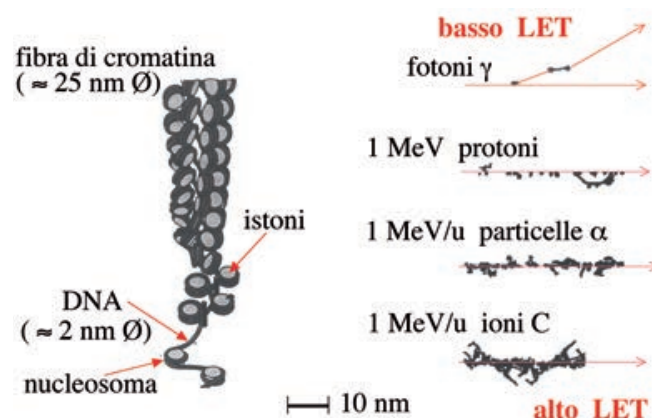

$1 \mathrm{MeV}$ protoni

$1 \mathrm{MeV} / \mathrm{u}$ particelle $\alpha$

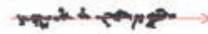

$1 \mathrm{MeV} / \mathrm{u}$ ioni $\mathrm{C}$

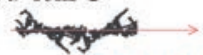

alto LET

Fig. 4 - Rilascio di energia di varie radiazioni (corpuscolari e non): a destra ogni punto rappresenta una interazione, a sinistra, sulla stessa scala dimensionale, è mostrata la doppia elica del DNA avvolta sulle strutture chiamate istoni. Il numero di interazioni per gli ioni carbonio (in basso a destra) sovrapposte al DNA sulla sinistra è tale da creare danni non riparabili al DNA.

\section{ACCELERATORI DI PARTICELLE}

Dalla fine degli anni '30 del secolo scorso sono stati sviluppati vari tipi di acceleratori di particelle circolari e lineari. L'accelerazione di particelle dotate di carica elettrica avviene impiegando campi elettrici in successione (acceleratori lineari) o attraversati numerose volte dalle particelle (acceleratori circolari).

In linea generale per ottenere fasci di particelle di elevata energia sono impiegati acceleratori circolari in cui le particelle nell'orbita circolare attraversano una regione dove un intenso campo elettrico imprime una accelerazione che viene ripetuta ad ogni passaggio fino a raggiungere l'energia richiesta ed essere poi espulsi con un campo magnetico deviatore verso la zona del bersaglio (sala di trattamento dove si trova il paziente). Le particelle sono trattenute nell'orbita circolare da campi magnetici: magneti curvanti e una sistema di accelerazione (cavità a radiofrequenza) costituiscono la base di tali acceleratori. 
Nel caso degli adroni (protoni e ioni) l'acceleratore di solito impiegato è chiamato sincrotrone. Un sistema di produzione delle particelle da accelerare (sorgente) invia "pacchetti" di particelle con una opportuna frequenza in un pre-acceleratore (di solito lineare) che con campi elettrici accelera ad una energia minima di ingresso nel sincrotrone in cui i pacchetti di particelle in orbita circolare sono accelerati da campi elettrici alternati in opportuni dispositivi (cavità a radiofrequenza). Ad ogni passaggio nella cavità il pacchetto incrementa la propria energia per cui, per permanere sempre nell'orbita circolare, il campo magnetico dei magneti curvanti viene aumentato, in contemporanea all'accelerazione. Il processo di accelerazione procede con numerosissime orbite, in tempi dell'ordine di frazione di secondo, fino all'energia richiesta.

I pacchetti di particelle sono composti da particelle della stessa carica elettrica tra le quali agisce quindi la repulsione coulombiana. Per evitare la disgregazione del pacchetto in brevissimo tempo, tra i magneti curvanti sono inseriti magneti focheggianti il pacchetto di particelle, mantenendolo compattato. Come detto, un campo magnetico devia il fascio di pacchetti una volta raggiunta l'energia desiderata inviandolo verso la sala del bersaglio tramite una successione di campi magnetici curvanti e focheggianti (linea di trasporto magnetica).

\section{Il Centro Nazionale di Adroterapia OnCologica}

Dopo circa 10 anni di progettazioni, proposte e richieste ai vari governi che si sono succeduti, a partire dal 2002 viene finanziata la costruzione del Centro Nazionale di Adroterapia Oncologica (CNAO) basato su un sincrotrone da $250 \mathrm{MeV}$ di energia massima per i protoni e con 3 sale di trattamento (Fig. 5). Il sistema è in grado di accelerare nella stessa ciambella (sotto vuoto spinto) sia i protoni che gli ioni carbonio. Quest'ultima importante caratteristica permette di irraggiare con protoni e poi con ioni senza che il paziente venga spostato e riposizionato. Il Centro è situato a Pavia in un terreno compreso tra gli IRCCS S. Matteo, la Fondazione Mondino e la Fondazione Maugeri. Al fine di evitare sprofondamenti e deformazioni dell'edificio a causa dell'elevato peso del cemento necessario, essendo il terreno alluvionale, l'acceleratore del Centro è stato costruito su una piattaforma di cemento armato spessa 2 metri che poggia su oltre 1500 palafitte costituite da cilindri di cemento armato alti 12 metri del diametro di 1,5 metri. 


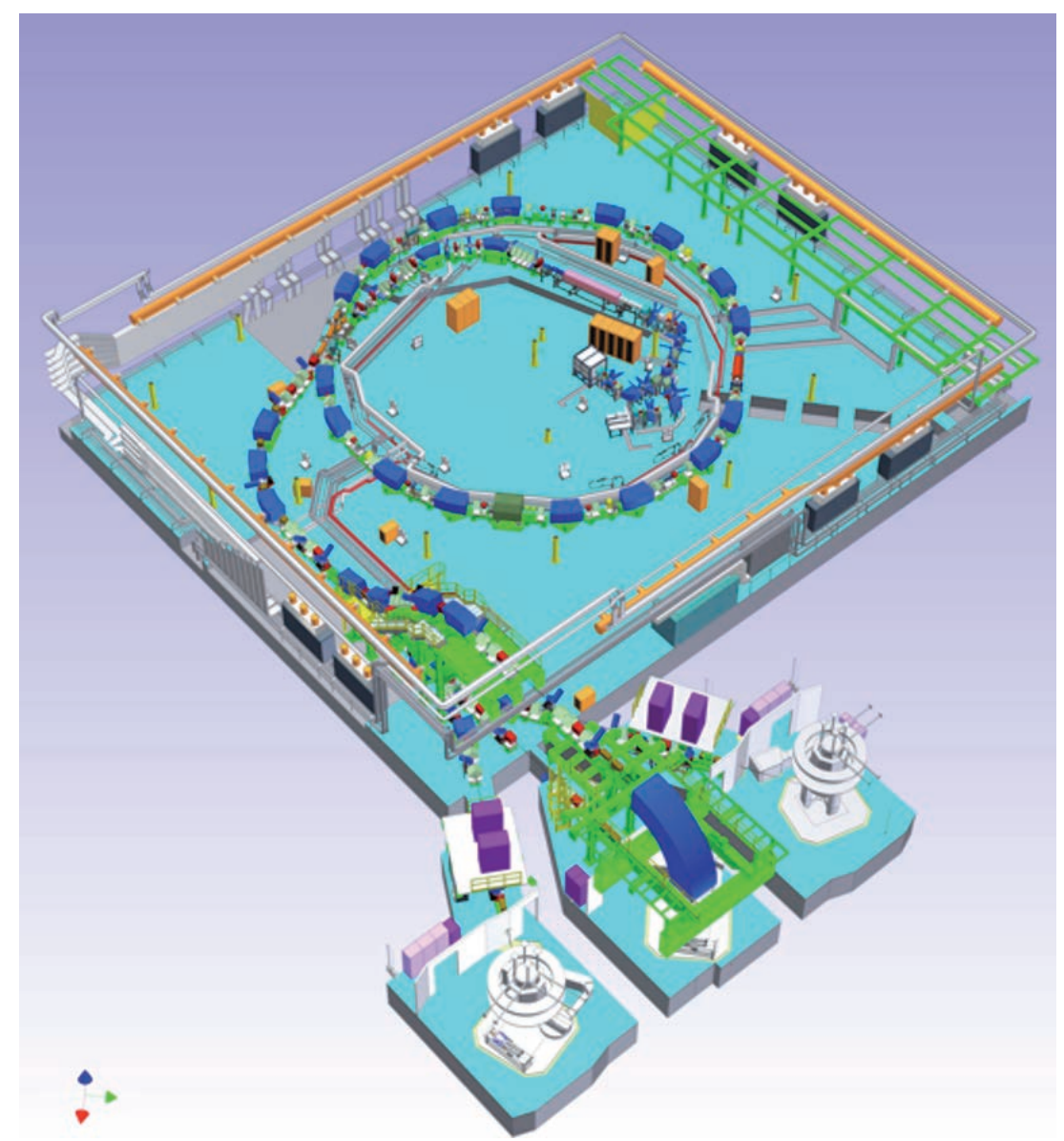

Fig. 5 - Il sincrotrone del CNAO con le 3 sale di trattamento. In blu sono i magneti curvanti, il cilindro rosso a destra nell' anello circolare è la cavità risonante di accelerazione, al centro il pre-acceleratore lineare in rosa e $i$ due cubi grigio-bianchi che contengono le sorgenti (di protoni e di ioni carbonio). La sala di trattamento centrale riceve dall'alto, tramite il magnete curvante blu, un fascio anche verticale. I lunghi cilindri arancioni ai lati sono il sistema di condizionamento della sala mentre le righe grigie al suolo contengono le tubature dell'acqua di raffreddamento dei numerosi magneti.

Il sincrotrone ha un diametro di circa 21 metri. Le rilevanti dimensioni sono determinate dalla massa dei protoni, 2000 volte più massicci degli elettroni, e degli ioni carbonio (di massa 24 mila volte quella dell'elettrone) e quindi altrettanto più difficili da curvare a parità 
di campi magnetici: infatti gli acceleratori di elettroni (che producono anche fasci di raggi X) nella radioterapia convenzionale sono lineari e hanno dimensioni di circa solo 1 o 2 metri.

Il sincrotrone è stato basato su magneti tradizionali soprattutto per motivi di sicurezza e stabilità nel funzionamento a scopi terapeutici, escludendo l'impiego di magneti superconduttori (di complessa manutenzione e non sicura affidabilità) che avrebbero permesso una drastica riduzione delle dimensioni dell'acceleratore. Delle tre sale di trattamento quella centrale, oltre che disporre di un fascio orizzontale, permette anche l'irraggiamento verticale tramite un sistema di trasporto magnetico del fascio che devia il fascio verso l'alto fino a farlo giungere verticalmente sul paziente. In Fig. 6 è mostrata una visione d'insieme del sincrotrone.

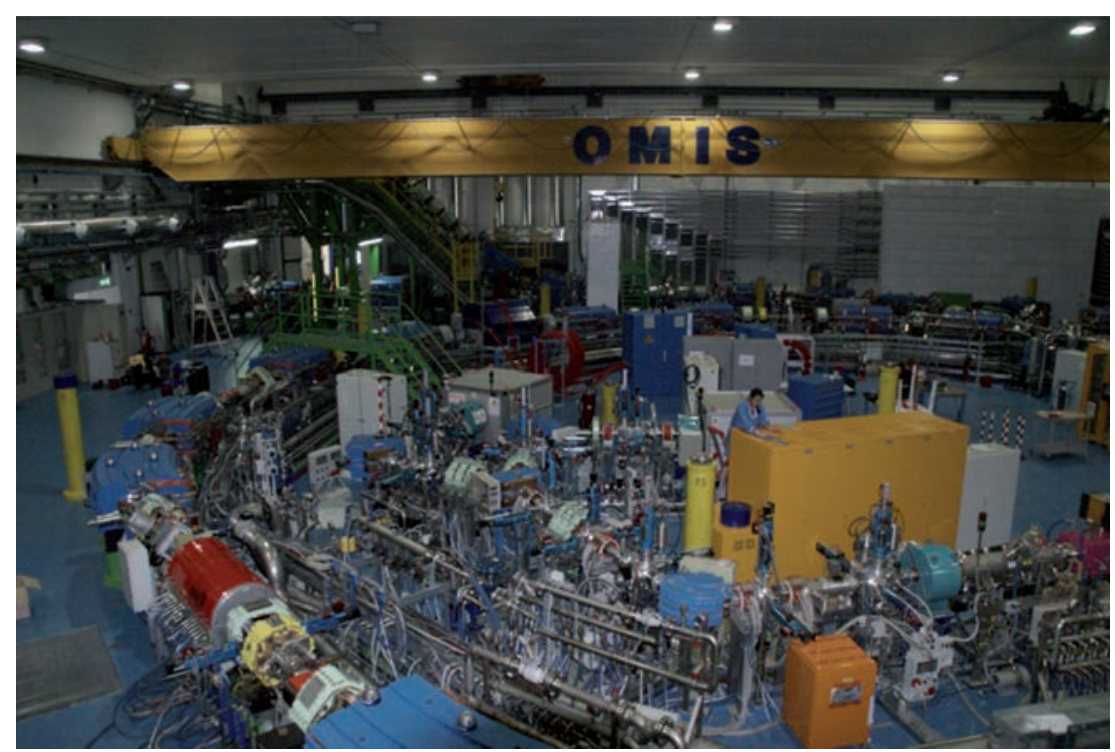

Fig. 6 - Visione d'insieme del sincrotrone.

La cavità risonante in cui avviene l'accelerazione è in rosso in basso a sinistra.

\section{TECNICHE DI POSIZIONAMENTO}

Come riferito in precedenza, la posizione del bersaglio (e quindi del paziente) deve avere una precisione millimetrica, non facile da ottenere e tenere sotto controllo per un organismo vitale. A questo scopo sono state applicate tecnologie di posizione estremamente accurate, derivate da atti- 
vità spaziali. Infatti il paziente viene cosparso nella zona cutanea da irraggiare (sotto la quale vi è il tumore da distruggere) con markers che riflettono la radiazione infrarossa e contenenti pallini di piombo, visibili nelle radiografie (nelle regioni capo-collo o torace o addome). Con radiografie e/o tomografie in raggi $\mathrm{X}$ si studia il volume da irraggiare avendo visibili anche i pallini di piombo contenuti nei markers: in questo modo si correlano le posizioni nelle 3 dimensioni dei pallini col volume del tumore. In un locale in prossimità della sala di trattamento il paziente viene disteso su un tavolo di carbonio, anch'esso dotato di markers, e posizionato, note la posizione dei markers sul proprio corpo e la posizione e forma del tumore, in riferimento ad alcuni markers sul tavolo. Il paziente viene posizionato seguendo le indicazioni del piano di trattamento e poi trasportato nell'adiacente sala di trattamento sul fascio dove telecamere digitali riprendono le immagini dei markers illuminati da radiazione infrarossa a 100 Hertz (100 volte al secondo). Le posizioni dei markers sul paziente e sul tavolo devono corrispondere a quelle richieste dal piano di trattamento entro l'accuratezza di circa 1 millimetro (le posizioni dei markers ottenute dalle immagini digitali infrarosse sono confrontate in tempo reale con quanto richiesto dal sistema di calcolo). Durante l'irraggiamento qualsiasi spostamento dei markers maggiore della precisione richiesta (ad esempio uno starnuto del paziente) causa l'interruzione istantanea del trattamento. Tutto ciò per la sicurezza del paziente, data la notevole efficacia distruttiva degli adroni. Il posizionamento per la sua accuratezza e importanza fondamentale dura alcune decine di minuti. Il tavolo su cui è posto il paziente è incastrato su un braccio robotico che su comando del calcolatore, lo sposta e lo orienta, secondo il piano di trattamento. In Fig. 7 è mostrata una sala di trattamento mentre in Fig. 8 è mostrata la disposizione e l'impiego dei markers nel caso di un tumore cerebrale (nella parte destra sono visibili le telecamere digitali e gli apparati radiografici per il controllo della posizione).

\section{TECNICHE DI IRRAGGIAMENTO}

Anche l'irraggiamento, eseguito seguendo il piano di trattamento con metodica attiva, è tecnologicamente complesso. Il fascio sottile (pencil beam) a una determinata energia, proveniente dall'acceleratore viene deviato da opportuni magneti lungo le coordinate in x e in y, esegue una scansione di una sezione del tumore distruggendola. Variando l'energia del fascio vengono distrutte via via le altre sezioni conformando la fettina 
da distruggere al contorno noto del tumore seguendo sempre le istruzioni del piano di trattamento (vedi Fig. 9). L'intera procedura di irraggiamento dura solo alcuni minuti. Quando il piano di trattamento prevede l'impiego non solo di protoni ma anche di ioni (ad esempio per distruggere la parte radio-resistente del tumore), il paziente non viene spostato mentre in tempi relativamente brevi si procede all'accensione della sorgente di ioni e alla accelerazione del nuovo fascio. Lungo la ciambella entro cui passano le particelle opportuni rivelatori permettono di conoscere e controllare l'intensità e la conformazione del fascio in tempo reale.

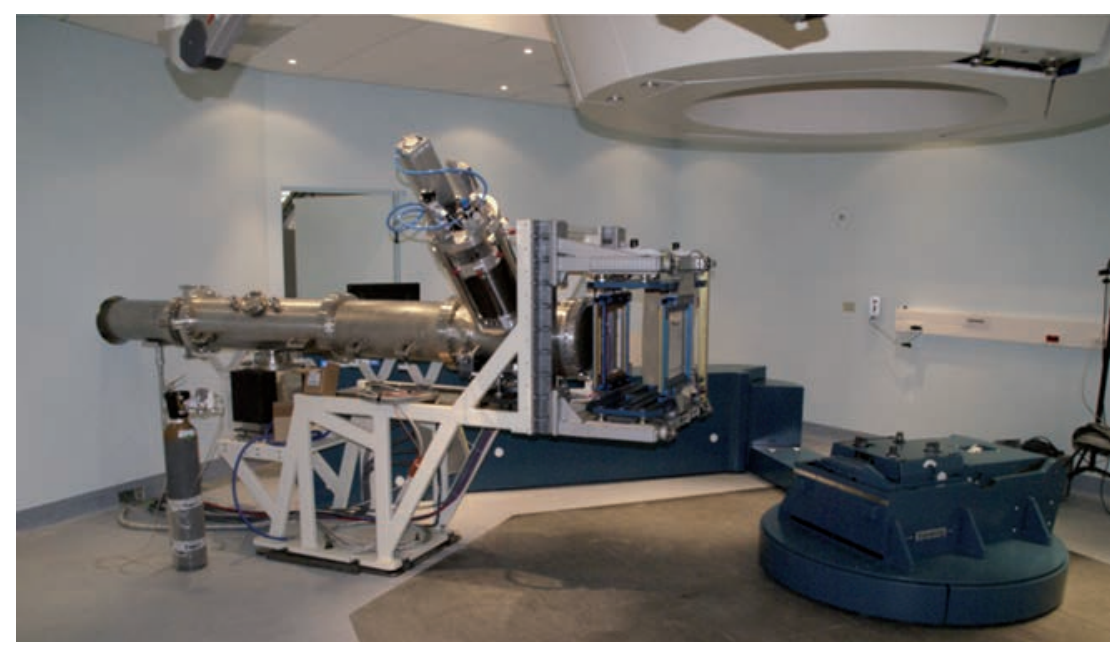

Fig. 7 - Sala di trattamento laterale in cui è visibile il lettino in carbonio. Dal rettangolo sulla destra esce il fascio.
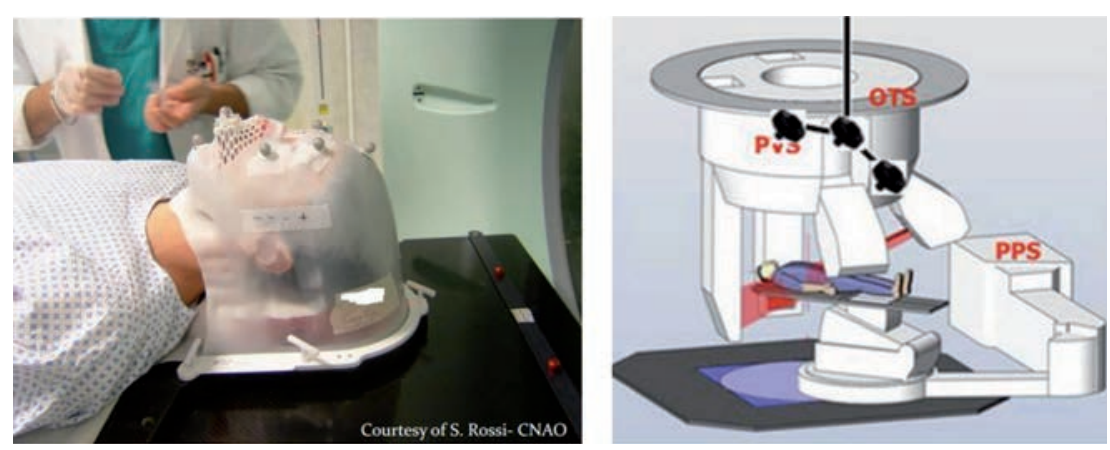

Fig. 8 - Posizionamento di un paziente (a sinistra) con i markers riflettenti. A destra sono mostrate le telecamere e le lampade ad infrarosso (PVS), 


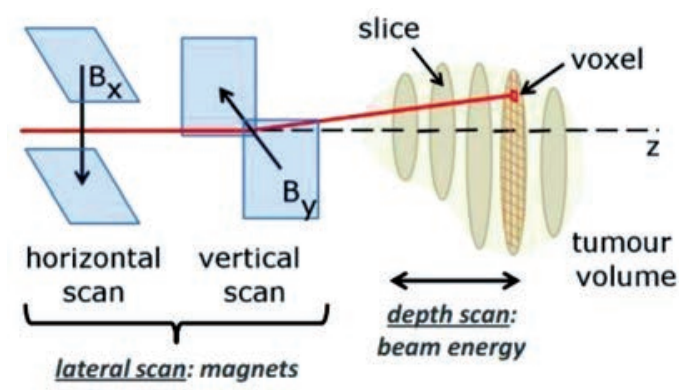

Fig. 9 - Schema di irraggiamento attivo tramite la scansione di una sezione dopo l'altra. Lo spostamento lungo la profondità z si ottiene variando l'energia del fascio.

\section{ConClusioni}

La radioterapia con adroni è iniziata al CNAO nel 2012 con irraggiamenti sperimentali di pazienti selezionati ed è divenuta parte del Servizio Sanitario Nazionale dal $1^{\circ}$ gennaio 2014. Allo stato attuale sono in funzione tutte le tre sale di trattamento, con il Centro in piena operatività terapeutica durante il giorno mentre nella notte proseguono le attività di ricerca scientifica sia tecnologica sia sullo studio degli effetti delle radiazioni sui vari tipi di cellule tumorali con lo scopo di migliorare l'efficacia dell'adroterapia. L'operatività del Centro è pertanto di 24 ore su 24 come avviene in tutti i centri di ricerca dotati di acceleratori, con interruzioni periodiche per manutenzione. In futuro si prevede di sviluppare un fascio destinato ad una sala di ricerca che opererà a livello internazionale. L'attività del Centro viene controllata da un Comitato Scientifico internazionale di cui fanno parte i maggiori esperti nel settore dell'adroterapia provenienti dal Giappone, dagli USA e dalla Germania, tutti Paesi ove sono sorti i principali centri di adroterapia nel mondo. 